\title{
Agrargeographie im Wandel
}

\section{Die «traditionelle» Agrargeographie}

Die Agrargeographie galt lange Zeit als Musterbeispiel einer anthropogen orientierten wissenschaftlichen Geographie. Die enge Verknüpfung von Raumbeobachtung und Raumanalyse führte zwangsläufig zur intensiven Befassung mit unterschiedlichen Nutzungsformen (1). Ein breites Spektrum anerkannter Autoren, angeführt von H.BERNHARD, H.RÜHL, H. ENGELBRECHT, L. WAIBEL, W. CREDNER, G. PFEIFER, W. MÜLLERWILLE, C.TROLL, E. OTREMBA usw. - um nur einige Namen zu nennen - trug besonders im deutschsprachigen Raum dazu bei, die Agrargeographie zur «klassischen» Forschungsdisziplin zu machen.

Die Agrargeographie war stets ein unumstrittener Bestandteil der Wirtschaftsgeographie. Besonders deutlich wurden in der morphogenetischen, dann aber auch in der funktionalen Phase vor und nach der Mitte dieses Jahrhunderts (2) wissenschaftliche Entwicklung, Arbeitsweisen und Zielsetzungen dieser Teildisziplinen erkennbar. Die Ablösung aus der rein

Gesamtheit des Informationsspektrums

Selektierte Informationen

Bewertung der Informationen

Raumwirksame Aktivitäten innerhalb der Grundfunktionen

Verortete Muster und Reichweiten

Entwurt: K. Ruppert Wearbellung. F. Eder ches institu

Abb. 1 Sozialgeographisches Raumsystem beziehungswissenschaftlichen, oft auf der Interpretation statistischer Daten beruhenden Konzeption führte besonders unter dem Einfluß ökonomischer Fragestellungen, die durchaus auch historisch-geographischen Überlegungen Platz ließen, zu stärker empirisch fundierten, kleinräumlichen Raumbeobachtungen und Kartierungen, deren hohe Bewertung besonders unter dem Einfluß CREDNERs um die Mitte des Jahrhunderts in Arbeiten zahlreicher geographischer Institute sichtbar wurde. Der von E. OTREMBA in den 60er Jahren herausgegebene "Atlas der deutschen Agrarlandschaft» spiegelte diesen Einfluß zum Teil noch zu einer Zeit, als die wissenschaftsgeschichtliche Entwicklung schon weiter fortgeschritten war, wider. Durch den Einbezug sozialgeographischer Fragestellung wurden aber auch in dieser Publikation bereits neue Forschungsansätze sichtbar. Von wenigen Ausnahmen abgesehen, waren die Arbeiten der damaligen Zeit aber weniger anwendungs- bzw. praxisorientiert. L. WAIBEL war sicher ein Sonderfall, wenn er von seinen Doktoranden verlangte, daß sie sich die Hände schmutzig machen, d. h. durch Mitarbeit im landwirtschaftlichen Betrieb die agrargeographischen Probleme besser kennenlernen sollten.

\section{Agrargeographie unter dem Einfluß sozialgeographischer Struktur- und ProzeBforschung}

Die frühen Arbeiten von H. BOBEK und w. CHRISTALLER können stellvertretend für andere die Entwicklung funktionaler Denkweisen in der Geographie verdeutlichen. Dieser Gesichtspunkt wird in der Agrargeographie in dem «Agrargeographischen Betrachtungssystem» H.CAROLS (3) erkennbar. In Fortentwicklung dieser funktionalen Denkweise suchten dann H.BOBEK und W. HARTKE mit ihren Mitarbeitern eine Anwort auf die Frage nach dem eigentlichen Gestalter, dem Funktionsträger im Agrarraum. Viele dieser Arbeiten wuchsen aus agrargeographischen

Karl Ruppert, Prof. Dr., Institut für Wirtschaftsgeographie Universität München Ludwigstr. 28, D-8000 München 22 
Fragestellungen heraus und suchten einen Ansatz zur Erfassung gruppen- und schichtspezifischer Verhaltensweisen als raumgestaltendes Element. Begünstigt durch die damals leichtere Erhebung grundlegender agrargeographischer Sachverhalte (Nutzungs-, Verwertungs- und Sozialsystem) und gefördert durch eine lange Forschungstradition, war es möglich, vor allem in kleinräumlichen Studien Beispiele für sozialgeographisch typische Verhaltensweisen in ihrer räumlichen Vielfalt darzustellen (4). Das Studium der sozialen Differenzierung innerhalb der Kulturlandschaft, gebunden an sozialgruppenspezifische Wertvorstellungen - Beispiel Sozialbrache -, belegte die Erkenntnis der Relativierung des Naturpotentials, ohne dessen Bedeutung zu unterschätzen. Die bei den Nutzungskartierungen oft übersehene Mehrdeutigkeit der Physiognomie und die daraus folgende Unsicherheit für die Analyse versuchte man durch die Methode der Sozialkartierung, die typischerweise im Bereich starker sozialgeographischer Differenzierung der Landwirtschaft in Süddeutschland entwickelt wurde (5), einzuengen. BLENCK, DEGE u. a. verfeinerten dieses Instrument später (6).

Im Zusammenhang mit der Ende der 50er bis in die 70er Jahre hinein geführten intensiven methodologischen Diskussion wurde als wichtiger Bestandteil der Konzeption der Sozialgeographie (7) ein Raumsystem entwickelt, das nicht mehr objekt-, sondern tätigkeitsorientiert war. Es versuchte, die Zusammenführung raumwirksamer Aktivitäten innerhalb sozialgeographischer Gruppen anstrebend, die agrargeographisch bestimmte Raumsituation mehreren grundfunktionalen Funktionsfeldern, insbesondere aber dem Bereich «Arbeit» zuzuordnen. Die Wahrnehmung der «objektiven» Faktoren sowie ihre gruppenspezifische Bewertung erhielten ihre feste Position in diesem Raumsystem.

Zum Verständnis der wissenschaftsgeschichtlichen Entwicklung erscheint der Hinweis nicht überflüssig, daß auch die veränderte gesamtgesellschaftliche Entwicklung nicht ohne Einfluß auf die wissenschaftlichen Forschungen blieb. So verwundert es nicht, daß agrargeographische Studien in den 60er Jahren nicht mehr so stark im Vordergrund standen wie vorher. Stadt- und bevölkerungsgeographische Arbeiten besetzen die "vorderen Ränge» in der geographischen Forschung. Endlich wurden auch praxisorientierte Fragestellungen in engem Kontakt zur Entwicklung der Raumplanung stärker beachtet. Zwar konnte das Studium des sich deutlich entwickelnden Urbanisierungsprozesses an der nach wie vor existierenden Bedeutung der Landbewirtschaftung nicht vorbeigehen, doch die bevorzugte Bearbeitung agrargeographischer Fragestellungen war nicht mehr gegeben. Die «soziale Dimension» der Fachwissenschaften (A.GEHLEN) wirkte sich unter der Dynamik des Industrialisierungsprozesses stärker in der Aufwertung anderer Teildisziplinen, z. B. der Industriegeographie, aus (DFG-Schwerpunktprogramm).
Dabei soll allerdings nicht übersehen werden, daß sich insbesondere in den sozialistischen Ländern Ostund Südosteuropas eine anwendungsorientierte Agrargeographie deutlicher behaupten konnte, wie u. a. Arbeiten von J. KOSTROWICKI, G. ENYEDI, W. ROUBITSCHEK usw. zeigen.

\section{Agrargeographie und postindustrielle Raumentwicklung}

Die gerade in den letzten Jahrzehnten oft «sprunghaft» fortschreitende Raumentwicklung, besonders in den Industrieländern, blieb zwar von der Agrargeographie nicht unbeachtet; eine entsprechende Ausweitung des Forschungsfeldes hat sich bisher aber noch nicht in genügender Weise gezeigt.

Aus gesamtgesellschaftlicher Perspektive macht sich in der Raumentwicklung besonders der Wandel von der flächengebundenen Agrargesellschaft zur standortorientierten Industrie- und zentrenorientierten Dienstleistungsgesellschaft bemerkbar. Parallel zur jüngsten Entwicklung hat sich seit den 70er Jahren unter dem Einfluß zahlreicher Urbanisierungsabläufe (8) auch ein neues Wertbewußtsein gegenüber den nichtbesiedelten Räumen entwickelt. Mehr und mehr setzt sich die Auffassung durch, daß «Freiräume» (9) nicht nur in der Nachbarschaft industriegewerblicher Zentren weit über ihr Produktionspotential hinaus von Bedeutung sind und einer pfleglichen Fürsorge bedürfen. Auch wenn man nicht der Auffassung huldigt, daß ökologische Maßstäbe allein über die Nutzung von Freiflächen entscheiden sollen, wird man diese heute nicht mehr nur als potentielles Produktionspotential der Agrarwirtschaft betrachten. Neue Aufgaben treten - getragen von veränderten Verhaltensweisen in unserer Gesellschaft - in Form von Nutzungsansprüchen an den Raum heran; oft werden multifunktionale Raumansprüche, aber auch Nutzungskonkurrenzen und Nutzungskonflikte sichtbar.

Von großer Bedeutung ist in diesem Zusammenhang die Entwicklung der Grundfunktion Freizeitverhalten, deren Raumbedeutsamkeit heute immer noch nicht in genügendem Ausmaß erkannt ist. Erst seit 1979 erhebt die Amtliche Statistik in der Bundesrepublik Deutschland mit bisher nicht übermäßigem Erfolg Flächen der Erholungsnutzung als eigene Nutzflächenkategorie. Flächenbilanzen werden aufgestellt (10), die Funktionszugehörigkeit von Nutzflächen wird neu überdacht. Veränderte Bewertungsmuster lassen einerseits bisher landwirtschaftlich genutzte Flächen uninteressant werden oder führen andererseits zur Mehrfach- ja Übernutzung. Eine Aufgabendifferenzierung landwirtschaftlicher Aktivitäten nach produktions- und landschaftspflegeorientierter Tätigkeit wird sichtbar. Freiräume werden nicht nur 


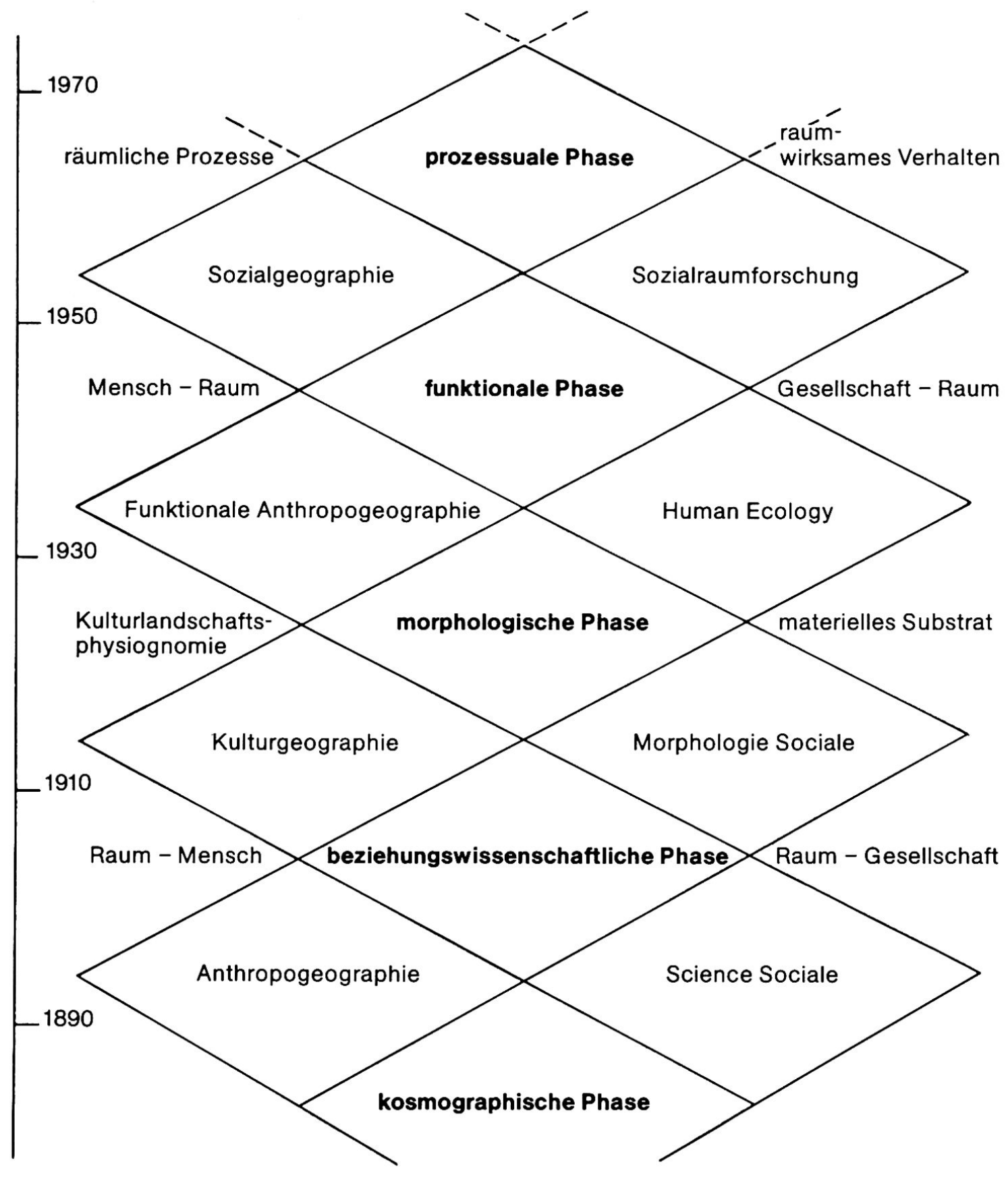

Abb.2 Übersicht über die Phasen anthropogeographischer und empirisch-soziologischer Wissenschaftsentwicklung (nach Thomale 1972)

genutzt, sie bedürfen auch der Pflege oder des Schutzes.

Mehr und mehr übt die Öffentliche Hand Einfluß auf die Raumnutzung aus. Eine schon nicht mehr überschaubare Vielfalt von Förder- und Pflegemaßnahmen in der Kulturlandschaft, aber auch die Ausweisung von Natur- und Landschaftsschutzgebieten nehmen immer stärker Einfluß auf Freiräume, die keineswegs in ihrer Gesamtheit als Agrarräume angesprochen werden können. Das Vordringen ökologischer Fragestellungen in der öffentlichen Diskussion wurde in der deutschen Geographie trotz ihrer "Urheberschaft» jahrelang übersehen. Nur selten ist es bekannt, daß C. TROLL bereits 1938 (11) den Begriff «Landschafts- 
ökologie» in die wissenschaftliche Diskussion eingeführt und in seinen Vorlesungen 1945/46 bereits den Begriff «Ökotop» benutzt hatte. Er wollte sich damit deutlich von der «Human Ecology» absetzen, die der Geograph H. H. BARRow 1922 (12) definierte. Erst nachdem in der Öffentlichkeit der Ruf nach ökologischer Forschung immer lauter wurde, gewann diese Fragestellung auch in der Geographie wieder breiteren Raum.

\section{Agrargeographie versus Geographie «ländlicher Räume»}

In den vergangenen Jahrzehnten wurden allmählich veränderte Forschungsschwerpunkte erkennbar. Es ergab sich die Notwendigkeit, agrargeographische Arbeiten stärker in die Problematik «ländlicher Räume» einzubetten. Auch wenn manchmal die Bedeutung der Landwirtschaft an ihrem Anteil am Bruttosozialprodukt oder an der Agrarerwerbsquote gemessen wurde, so war auch in industrialisierten oder stärker tertiär geprägten Bereichen ihre Bedeutung für die Gestaltung der Raumstrukturen unübersehbar. In dem Maße wie zum Beispiel die Bedeutung der Nebenerwerbsbetriebe gegenüber den Haupterwerbsbetrieben anwuchs, wie früher «rein» bäuerliche Siedlungen nur noch einen kleinen Teil landwirtschaftlicher Bevölkerung aufwiesen und die außerlandwirtschaftliche Wohn- bzw. Arbeitsfunktion zunahm, mußte aber der agrargeographische Betrachtungsrahmen den veränderten Einflußfaktoren der Raumorganisation Rechnung tragen. Agritourismus, industriemäßige Produktion von Nahrungsmitteln, Sozialfunktion der Landwirtschaft, Landbewirtschaftung ohne Agrarproduktion wurden zu Begriffen, die neue Entwicklungslinien aufzeigten. A.PIERSMA sah sich 1971 (13) veranlaßt, unter dem Titel "Agrarische geografie versus rurale geografie» auf den eingetretenen Wandel hinzuweisen. Die Agrargeographie sollte stärker in den Kontext und die Synthese «ländlicher Räume» einbezogen werden, d. h. aber auch, daß sie stärker außerlandwirtschaftliche Einflußfaktoren berücksichtigen mußte.

Gerade in den Industrieländern bildeten sich weitreichende Stadt-Land-Kontinua anstelle des früheren Stadt-Land-Gegensatzes. Neue Formen der Multifunktionalität der Raumnutzung, die Entfaltung des Einflusses weitreichender Urbanitätsgradienten waren nicht mehr aus dem agrarischen Wirkungsgefüge auszuschalten. Der Terminus «ländliche Räume» wurde im Titel überaus zahlreicher Publikationen auch außerhalb der Geographie sichtbar, die «Regionalisierung des Denkens» machte in der Öffentlichkeit beträchtliche Fortschritte. Gleichzeitig wurde aber auch ein gewisses «Problembewußtsein» gegenüber dieser von der Raumplanung neu entdeckten Gebiets- kategorie geweckt, verbargen sich doch ganz unterschiedliche Begriffsinhalte, vom hoch bewerteten Freizeitraum bis zum abwanderungsgefährdeten Mittelgebirgsbereich, von Gebieten hochproduktiver Landwirtschaft bis zur Zone der «urbanen Landbewirtschaftung» hinter der so einfachen, aber so praktikablen Vokabel. Nach wie vor aber bleibt der Begriff «ländlicher Raum» schillernd; auch alle Versuche, von sogenannten "peripheren» Räumen zu sprechen, führten nicht weiter.

\section{Agrargeographie auf neuen Wegen?}

Geht man von der auch im öffentlichen Bewußtsein steigenden Wertschätzung der Freiräume aus, könnte man sich die Frage vorlegen, ob wir auch von einer Aufwertung der Agrargeographie als wissenschaftlicher Disziplin, die sich mit Freiräumen beschäftigt, stehen. Die zahlreichen Arbeiten agrargeographischen Inhaits aus dem Bereich der Entwicklungsländer, die Neuauflage des im deutlichen Kontakt zur Agrarökonomie stehenden Lehrbuchs von ANDREAE (14), das Erscheinen eines Bandes "Agrargeographie» in der Reihe «Das Geographische Seminar» (15) und gar eine seit 1983 neu erscheinende «Zeitschrift für Agrargeographie» neben den seit vielen Jahren von w. ROUBITSCHEK herausgegebenen, bei uns leider viel zu wenig bekannten «Mitteilungen für Agrargeographie, landwirtschaftliche Regionalplanung und ausländische Landwirtschaft» könnten eine positive Beantwortung dieser Frage nahelegen.

Dennoch scheint Vorsicht am Platze. Wenn A.AR NOLD, einer der Herausgeber der neuen Zeitschrift, im gewissermaßen programmatischen ersten Artikel unter Wiederverwendung des Titels der wegweisenden Veröffentlichung von H.BERNHARD die Agrargeographie definiert «als die Wissenschaft von der räumlichen Ordnung und räumlichen Organisation der Landwirtschaft» (16) und in Anlehnung an ältere Autoren «die verwandten Zweige des primären Wirtschaftssektors», d.h. Jagd, Fischerei und sogar Forstwirtschaft, aus der Agrargeographie ausschließt, dann scheint mir diese Fassung der Agrargeographie schon deshalb zu eng, weil z. B. alle Betriebe, für die Waldbesitz fester Einkommens- und Betriebsbestandteil ist, an den Rand der Betrachtung gerückt werden. Später folgende Artikel in der Zeitschrift zeigen im übrigen, daß diese inhaltliche Einengung gar nicht durchgehalten werden kann.

Eine in anderer Hinsicht zu enge Auffassung finden wir auch bei SIcK (15, S. 7), wo im wichtigen Abschnitt «Definition und Ziele» zu lesen ist:

«Die Agrargeographie ist eine Raumwissenschaft, und ihr Forschungsgegenstand ist somit die durch die Landwirtschaft gestaltete Erdoberfläche. Die Land- 
wirtschaft wiederum kann als «Bewirtschaftung des Bodens zur Gewinnung pflanzlicher und tierischer Produkte für die Bedarfsdeckung der Menschheit» definiert werden (F. AEREBOE 1923)».

Landbewirtschaftung ohne Nahrungsmittelproduktion, wie sie heute bereits von Landwirten unter dem Einfluß des Freizeitverhaltens weiter Bevölkerungskreisen praktiziert wird, scheidet damit aus der Betrachtung aus, ebenso die Problematik, die mit der «nichtproduktiven» Dienstleistungsfunktion einer modernen Landwirtschaft verbunden ist.

Derartige Perspektiven lassen die Frage nach dem "Aufwind» für die Agrargeographie schon skeptischer beurteilen. Die Aufgabenstellung der Landwirtschaft ist schon längst über die Produktionsfunktion hinausgewachsen. Schon seit 1970 wird im «Gesetz zur Förderung der bayerischen Landwirtschaft» Art. 1, Abs. 1, festgestellt, daß die Erhaltung der Kulturlandschaft eine Aufgabe der Landwirtschaft ist, und sehr deutlich stellte ebenfalls vor mehr als einem Jahrzehnt das "Landwirtschafts- und Landeskulturgesetz von Baden-Württemberg» 1972 fest (Abs. 1 §), daß Forstund Landwirtschaft der Allgemeinheit durch «Gestaltung und Pflege der Kultur- und Erholungslandschaft» zu dienen hat. In keinem Fall wird die Produktion von Nahrungsmitteln als Voraussetzung zur Erfüllung dieser Aufgaben verlangt.

Nein, keine enge Beschränkung, sondern eine Öffnung der Forschungsperspektiven über die traditionellen Fragestellungen der Nutzungs-, Betriebs- und Verwertungsstrukturen und der entsprechenden Prozeßabläufe hinaus muß erreicht werden. Probleme wie z. B.

- Landbewirtschaftung ohne Agrarproduktion

- Agrarraum als Landreserve

- Einfluß der Fördermaßnahmen auf die Landbewirtschaftung im nationalen und internationalen Bezug

- Bedeutung der Ausweisung von Schutzgebieten, dadurch veranlaßte Einschränkung der Dispositionsmöglichkeit für landwirtschaftliche Betriebe

- Wahrnehmung von Umweltschutzaufgaben und Beeinflussung der Umwelt durch die Landwirtschaft

- Bedeutung der Agrarwirtschaft für die Bereitstellung natürlicher Ressourcen

- multifunktionale Flächennutzung und Flächennutzungskonkurrenzen

- Dienstleistungscharakter der Landwirtschaft usw.

sind gerade im europäischen Bereich auch in den agrargeographischen Arbeiten aufzugreifen. Diese Liste, die nur wenige, herausgegriffene Aspekte enthält, ließe sich unschwer erweitern.

Auch in engem Kontakt zu der sich mehr und mehr etablierenden Freiraumforschung hat eine "offene» Agrargeographie, wie sie auch das sozialgeographische Raumsystem durch seine Zentrierung raum- wirksamer Aktivitäten in der sozialgeographischen Gruppe nahelegt, zu stehen. Dann bleibt immer noch genügend Raum, für die auch in den Industrieländern nach wie vor nicht unbedeutende geographische Erforschung der Nahrungsmittelproduktion. Auf diese Weise eröffnen sich zusätzlich Forschungsfelder, die überdies die Bedeutung der wissenschaftlichen Forschung für unsere Gesellschaft erneut dokumentieren können.

\section{Anmerkungen:}

1. K. RUPPERT (Hrsg.): Agrargeographie, Wege der Forschung, Band 171, Darmstadt 1973

2. E. THOMALE: Sozialgeographie, eine Disziplingeschichtliche Untersuchung zur Entwicklung der Anthropogeographie, Marburger Geographische Schriften 53/1972

3. H.CAROL: Das agrargeographische Betrachtungssystem, Geographica Helvetica 1952, S. $17 \mathrm{ff}$.

4. v. V. Sozialgeográphie, Das Geographische Seminar, Braunschweig 1977

5. vgl. dazu die Literaturzusammenstellung in (1)

6. K.RUPPERT: Spalt, ein methodischer Beitrag zum Studium der Agrarlandschaft mit Hilfe der kleinräumlichen Nutzflächenund Sozialkartierung und zur Geographie des Hopfenbaues, München, Geogr. Hefte 14/1958

J.BLENCK: Die Insel Reichenau, eine agrargeographische Untersuchung, Heidelberger Geographische Arbeiten, 33/1971 E.DEGE: Filsen und Osterspai, Arbeiten zur Rheinischen Landeskunde 36/1973

7. vgl. (4), S. 26

8. R. PAESLER: Urbanisierung als sozialgeographischer Prozeß, MSSW 12/1976

9. G.BÄUERLE: Der Freiraum als räumliches Wertobjekt, München: Minerva-Publikation 1984

10. E.BUCHHOFER: Aktuelle Entwicklungen der Flächennutzung in Mitteleuropa, Marburger Geographische Schriften, 88/1982

P.LINTNER: Flächennutzung und Flächennutzungswandel in Bayern, Dissertation, WGI München 1984

11. C. TROLL: Luftbildplan und ökologische Bodenforschung, Z. d. Ges. f. Erdkunde zu Berlin 1939

12. H. H. BARROW: Geography as Human Ecology, Ann. of Ass. Am. Geographers 13/1973, S. $1 \mathrm{ff}$.

13. A. PIERSMA: Agrarische geografie versus rurale geografie, Geogr. Tijdschrift 1/1971, S. 25ff.

14. B. ANDREAE: Agrargeographie, Berlin 1977

15. W.-D. SICK: Agrargeographie, Das Geographische Seminar, Braunschweig 1983

16. A.ARNOLD: Die Agrargeographie als wissenschaftliche Disziplin, Z. f. Agrargeographie 1/1983, S. 3ff. 